УДК 54.01+544.2

\title{
СИНТЕЗ СУБМИКРОЧАСТИЦ SrO и ВаО МЕТОДОМ ТЕРМИЧЕСКОГО РАЗЛОЖЕНИЯ АЭРОЗОЛЕЙ
}

\author{
(C) 2018 А. И. Горшков, Е. Н. Грибанов, Э. Р. Оскотская \\ Орловский государственный университет имени И. С. Тургенева \\ ул. Комсомольская, 95, 302026 Орёл, Россия \\ e-mail: gribanovEN@gmail.com
}

Поступила в редакцию 06.11.2018

\begin{abstract}
Аннотация. В работе получены субмикрочастицы оксида стронция и оксида бария методом термического разложения аэрозолей. Природа синтезируемых соединений доказана методом ИК-спектроскопии. Определены характерные линейные размеры и изучена морфология поверхности частиц методом атомно-силовой микроскопии. Показано, что при пиролизе происходит дробление капли жидкости, а образующаяся твердая фаза представляет собой полые сферы. На основе спектров диффузного отражения определена оптическая ширина запрещенной зоны объектов. Полученные данные представляют интерес при дальнейшем изучении свойств субмикро- и наноструктур на основе $\mathrm{SrO}$ и $\mathrm{BaO}$, а также при синтезе новых функциональных материалов, обладающих улучшенным комплексом свойств.
\end{abstract}

Ключевые слова: оксид стронция, оксид бария, ширина запрещенной зоны, пиролиз.

DOI: https://doi.org/10.17308/kcmf.2018.20/631

\section{ВВЕДЕНИЕ}

Тонкодисперсные порошки оксида стронция $(\mathrm{SrO})$ и оксида бария $(\mathrm{BaO})$ используют при получении композиционных материалов и покрытий с широким спектром электрофизических и оптических свойств. Они находят применение при синтезе высокотемпературной керамики, в качестве радиопрозрачных конструкционных материалов, а также материалов для энергетики, машиностроения, электрохимии, катализа и т. д. [1-5]. Требования к современной техники и уровень развития технологий создают необходимость в улучшении свойств и характеристик функциональных материалов. Одним из способов решения поставленной проблемы является переход от массивного тела к низкоразмерным структурам субмикро- и нанометрового размера. Данный переход сопровождается, как правило, скачкообразным изменением свойств вещества [6], что может найти практическое применение при понижении температуры спекания керамики на основе оксидов стронция и бария, обеспечить объемную однородность свойств керамических элементов, повысить эффективность каталитических систем, служить основой производства нелинейнооптических материалов и другое. Среди методов синтеза оксидных соединений металлов, позволяющих существенно снизить температуру их образования, получать продукты в виде ультрадисперсных порошков хорошо зарекомендовал себя метод термического разложения аэрозолей в потоке высокотемпературного газообразного теплоносителя [7-9]. Данный способ обеспечивает образование оксидов металлов заданного химического состава, однородность распределения по размеру частиц и другим текстурным характеристикам.

Целью настоящей работы явилось получение субмикрочастиц $\mathrm{SrO}$ и $\mathrm{BaO}$ методом термического пиролиза аэрозолей, содержащих соответствующие прекурсоры, а также определение некоторых текстурных и энергетических характеристик, полученных низкоразмерных структур.

\section{ЭКСПЕРИМЕНТАЛЬНАЯ ЧАСТЬ}

Маточные водные растворы прекурсоров $\left(\mathrm{Ba}\left(\mathrm{NO}_{3}\right)_{2}\right.$ и $\operatorname{Sr}\left(\mathrm{NO}_{3}\right)_{2}$, (ч. д. а.) с концентрацией $0.5 \mathrm{M}$ готовили растворением точной навески соли в бидистилированной воде. Выбор природы прекурсоров связан с возможностью дальнейшего применения относительно мягкого режима их термообработки, доступностью и относительно низкой стоимостью при реализации технологических 
процессов. Рабочие водные растворы $\mathrm{Ba}\left(\mathrm{NO}_{3}\right)_{2}$ и $\mathrm{Sr}\left(\mathrm{NO}_{3}\right)_{2}$ соответствующей концентрации готовили разбавлением исходного маточного раствора непосредственно перед экспериментом.

Основные принципы получения частиц методом термического разложения аэрозолей описаны в [7]. В настоящей работе водный раствор нитрата соответствующего металла $\left(\mathrm{n} \cdot 10^{-3}-\mathrm{n} \cdot 10^{-1} \mathrm{M}\right)$ переводили с помощью компрессорного распылителя в состояние аэрозоля (диаметр капли $4 \pm 1$ мкм), который с газом-носителем воздухом (скорость потока $\sim 0.25 \mathrm{~m} / \mathrm{c}$ ) переносится в цилиндрический кварцевый реактор (длина 210 мм, внутренний диаметр 25 мм), нагретый до постоянной температуры $750 \pm 2{ }^{\circ} \mathrm{C}$. В реакторе происходит термолиз и оседание продуктов реакции на подложку $\left(620 \pm 2{ }^{\circ} \mathrm{C}\right)$, в качестве которой выступал алюминий (А99), предварительно подготовленный по методике [10].

Морфологию поверхности частиц и их размер определяли методом сканирующей зондовой микроскопии (микроскоп СММ-2000). Использовали режим атомно-силовой микроскопии (АСM). Сканирование проводили кантилевером фирмы Veесо марки MSCT-AUNM с длинной балки 180 мкм, жесткостью $0.05 \mathrm{H} /$ м и радиусом острия иглы >300 А̊. Обработка и количественный анализ АСМ изображений осуществляли с помощью программы Scan Master for SMM-2000.

ИК-спектры регистрировали в области 4000$450 \mathrm{~cm}^{-1}$ (ИК-фурье спектрометр ФСМ 2202) путем таблетирования с $\mathrm{KBr}$. Спектры диффузного отражения (СДО) получены на спектрофотометре

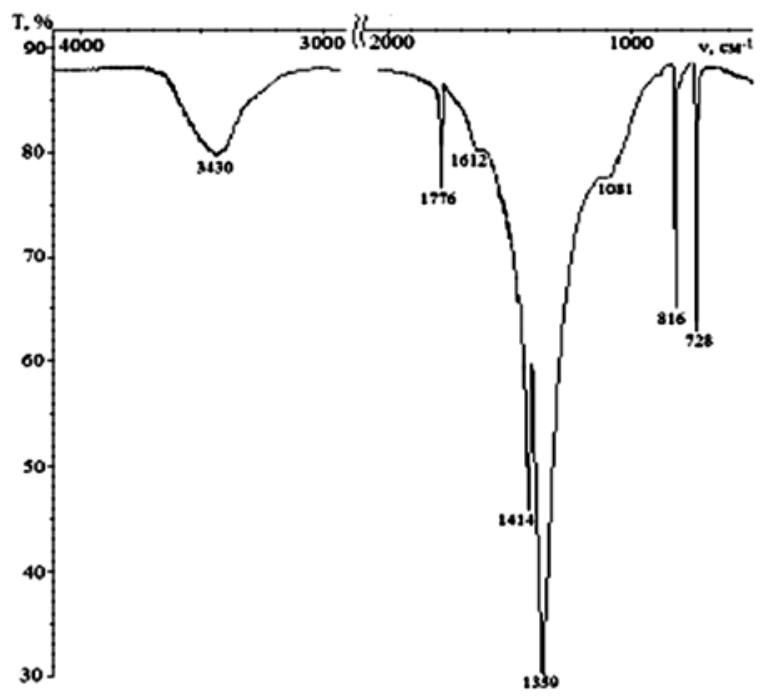

СФ-56 с приставкой диффузного отражения ПДО-6 (угол падения $8^{\circ}$ ).

\section{РЕЗУЛЬТАТЫ И ИХ ОБСУЖДЕНИЕ}

В условиях температурного режима, применяемого в работе, реакция разложения $\mathrm{Ba}\left(\mathrm{NO}_{3}\right)_{2}$ и $\mathrm{Sr}\left(\mathrm{NO}_{3}\right)_{2}$ протекает по схеме [11]:

$2 \mathrm{Me}\left(\mathrm{NO}_{3}\right)_{2}=2 \mathrm{MeO}+4 \mathrm{NO}_{2}+\mathrm{O}_{2}$, где $\mathrm{Me}-\mathrm{Sr}, \mathrm{Ba}$.

Идентификацию природы образующихся соединений, проводили путем сравнения их полос поглощения (ПП) в ИК-спектрах (рис. 1) с характеристическими ПП из литературных данных [1214]. Так полоса поглощения при $1414 \mathrm{~cm}^{-1}$ соответствует валентным колебаниям связи Ва-О, а ПП с частотой $1440 \mathrm{~cm}^{-1}$ валентным колебаниям связи $\mathrm{Sr}-\mathrm{O}$. Полосы поглощения при 816 и $728 \mathrm{~cm}^{-1}, 816$ и $736 \mathrm{~cm}^{-1}$ можно отнести, соответственно, к деформационным колебаниям связей $\mathrm{Ba}-\mathrm{O}$ и $\mathrm{Sr}-\mathrm{O}$. Полосы поглощения при 3430 и $1612 \mathrm{~cm}^{-1}, 3488$ и $1637 \mathrm{~cm}^{-1}$ можно отнести к валентным и деформационным колебаниям гидроксильных групп, связанных с атомами бария и стронция соответственно. При анализе ИК-спектров нами обнаружено сужение характеристических полос поглощения частиц $\mathrm{SrO}$ и $\mathrm{BaO}$ при уменьшении их диаметpa, что можно объяснить проявлением размерного эффекта.

Интенсивность процессов, протекающих в гетерогенной системе «твердое тело - среда», зависит от текстурных характеристик частиц - их размера, формы, состояния поверхности. Это связано с тем, что с уменьшением размера объекта, увели-

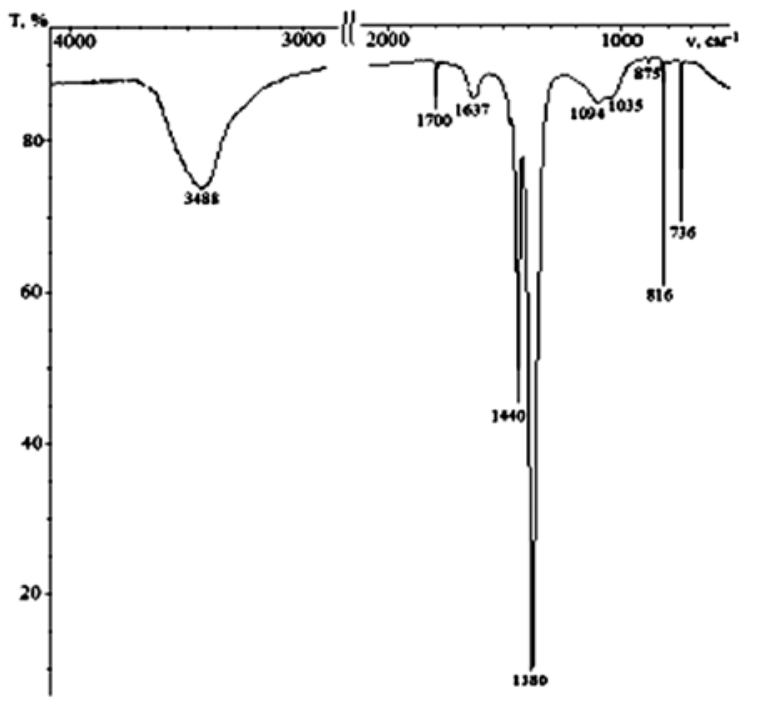

$a$

Рис. 1. ИК-спектр $\mathrm{BaO}(a)$ и $\mathrm{SrO}(b)$

$b$

[Fig. 1. IR-spectrum of $\mathrm{BaO}(a)$ and $\mathrm{SrO}(b)$ ] 
чивается его удельная площадь поверхности и избыточная свободная энергия, что приводит к изменению физико-химических характеристик и росту активности гетерогенной системы в процессе ускорения химических реакций. Типичное АСМ-изображение поверхности подложки с напыленными на неё частицами представлено на рис. 2. Анализ полученных АСМ-кадров показывает, что образующиеся частицы имеют форму близкую к сферической. Оксид бария представлен, как правило, в виде отдельных частиц, в то время как оксид стронция в виде их агломератов. Зависимость среднего диаметра частиц от исходной концентрации прекурсора приведена в табл. 1. Оценить теоретически ожидаемый диаметр синтезируемых структур можно по формуле:

$$
d=2\left[\frac{3 V_{\text {част }}}{4 \pi}\right]^{1 / 3},
$$

где $V_{\text {част }}=m / \rho$ ( $m$ - масса оксида металла с учетом стехиометрии уравнения разложения его нитрата, $\rho$ - плотность $\mathrm{BaO}$ или $\mathrm{SrO})$.

Рассчитанный таким образом диаметр частиц $\mathrm{BaO}$ и $\mathrm{SrO}$ не согласуется с реально наблюдаемым в эксперименте, что можно объяснить протеканием в реакторе процессов, связанных с дроблением капли при её нагреве и формированием твердой фазы не только в виде сплошной, но и полой частицы $[15,16]$.

В зависимости от соотношения объемов жидкости $\left(V_{\text {жид }}\right)$ и включения $\left(V_{\text {тв }}\right)$ существует три режима распада неоднородной капли жидкости при её интенсивном высокотемпературном нагреве [15].
Таблица 1. Геометрические и энергетические характеристики субмикрочастиц $\mathrm{SrO}$ и $\mathrm{BaO}$

[Table 1. Geometric and energy characteristics of $\mathrm{SrO}$ and $\mathrm{BaO}$ submicroparticles]

\begin{tabular}{|c|c|c|}
\hline $\begin{array}{c}\text { Природа оксида, исходная } \\
\text { концентрация прекурсора } \\
\text { [The nature of the oxide, the initial } \\
\text { concentration of the precursor] }\end{array}$ & $d_{\text {aver, }}, \mathrm{nm}$ & $E_{g}, \mathrm{eV}$ \\
\hline $\mathrm{BaO}, 1 \cdot 10^{-1} \mathrm{M}$ & $160 \pm 20$ & 2.95 \\
\hline $\mathrm{BaO}, 2 \cdot 10^{-2} \mathrm{M}$ & $115 \pm 30$ & 3.16 \\
\hline $\mathrm{SrO}, 3 \cdot 10^{-2} \mathrm{M}$ & $560 \pm 50$ & 3.15 \\
\hline $\mathrm{SrO}, 6 \cdot 10^{-3} \mathrm{M}$ & $150 \pm 20$ & 3.19 \\
\hline
\end{tabular}

Первый режим - распад на несколько (3-5) крупных фрагментов, реализуемый при $V_{\text {жид }}>V_{\text {тв}}$, второй - наблюдается в системе при отношении $V$ жид к $V_{\text {тв }}$ в диапазоне равном 1-2 и приводит к образованию 5-10 фрагментов, третий - совокупность множества (вплоть до нескольких сотен) мелких капель при $V_{\text {жид }}<V_{\text {тв }}$. В качестве объема включения использовали объем нитрата соответствующей соли. Для изучаемых нами систем $V_{\text {жид }} / V_{\text {тв }}$ составляет $\sim \mathrm{n} \cdot 10^{2}-\mathrm{n} \cdot 10^{3}$, т. е. преобладает первый тип дробления и каждая капля аэрозоля в реакторе распадается на 3-5 частей.

Формирование морфологии частиц оксидов стронция и бария происходит на стадии испарения капли, которое сопровождается одновременным протеканием следующих процессов: изменение температуры капли при нагревании, испарение растворителя с её поверхности, диффузия соли по направлению к центру капли, кристаллизация соли
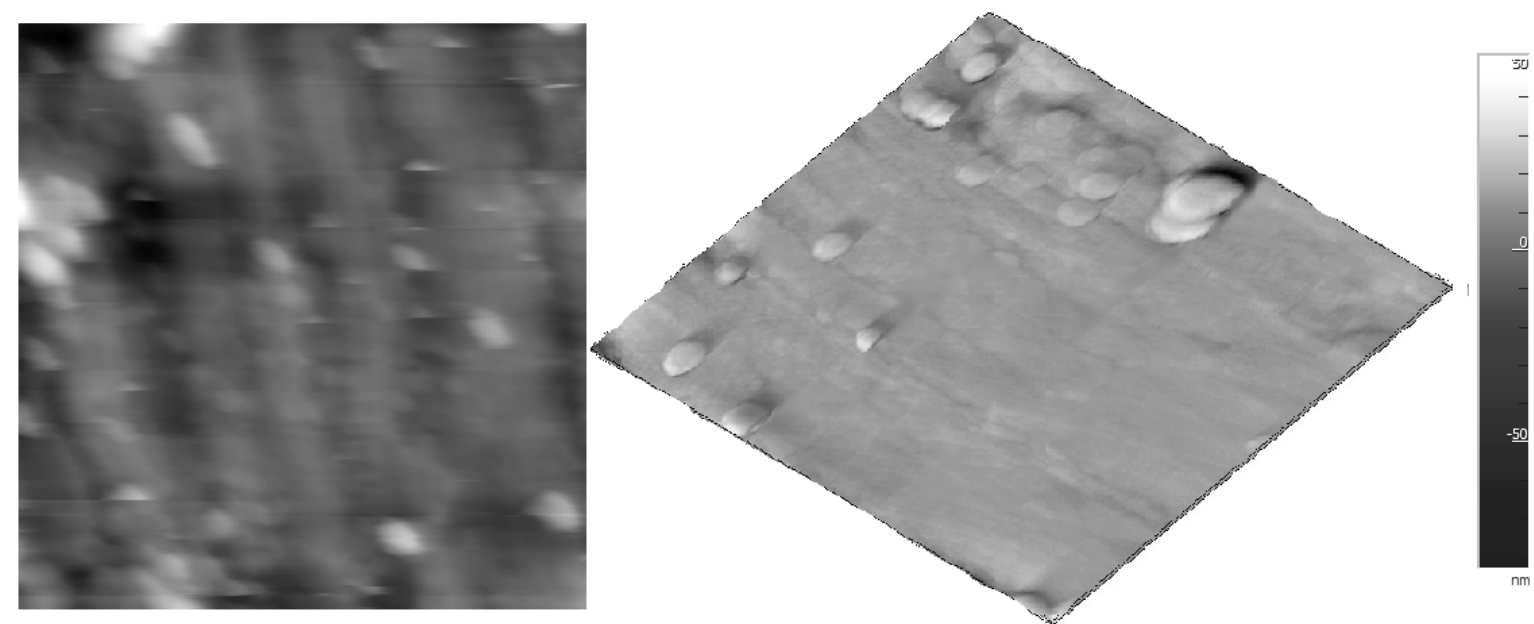

Рис. 2. Типичное АCM-изображение поверхности подложки после напыления на нее частиц $\mathrm{BaO}$ в $2 \mathrm{D}$ и $3 \mathrm{D}$ виде, размер изображения 2.742 .7 мкм, $C_{\mathrm{Ba}(\mathrm{NO}) 2}=2 \cdot 10^{-2} \mathrm{M}$

[Fig. 2. Typical AFM image of the substrate surface after deposition of $\mathrm{BaO}$ particles on it in $2 \mathrm{D}$ and $3 \mathrm{D}$ view, image size $\left.2.7 \mathrm{Ч} 2.7 \mu \mathrm{m}, C_{\mathrm{Ba}(\mathrm{NO} 3) 2}=2 \cdot 10^{-2} \mathrm{M}\right]$ 
и удаление оставшегося растворителя (обезвоживание капли). Безразмерным параметром $(K)$, определяющим структуру (морфологию) частицы, является отношение характерных времен диффузии и испарения: $K=t_{\text {диф }} / t_{\text {исп }}$. Эмпирически установлено [16], что при значениях $K<\sim 0.75$ диффузионные процессы идут быстрее процессов испарения, и образуются сплошные частицы, а при $K>\sim 0.75$ пустотелые.

Характерное время диффузии соли в объеме капли рассчитывали по формуле [17]:

$$
t_{\text {диф }}=R_{0}^{2} / D,
$$

где $R_{0}$ - начальный радиус капли, $D$ - коэффициент диффузии.

Время испарения жидкости с поверхности капли находили из модели приведенной пленки для процесса испарения [18]:

$$
t_{\text {исп. }}=\frac{2 \rho R_{0}^{2} C_{v}}{\lambda_{g} N u \ln (1+B)},
$$

где $B=C_{\mathrm{v}}\left(T_{e}-T_{\mathrm{v}}\right) / L-$ число Сполдинга; $N u$ - число Нуссельта, $\lambda_{g}-$ коэффициент теплопроводности газа при температуре кипения, $C_{\mathrm{v}}$ - удельная теплоемкость паров растворителя при постоянном объеме, $L$ - удельная теплота испарения, $T_{e}$ - температура газообразного теплоносителя, $T_{\mathrm{v}}-$ температура равновесного испарения (кипения).

При формировании фазы оксида стронция и оксида бария в результате термического разложения капли аэрозоля коэффициент $K$ составляет $\sim \mathrm{n} \cdot 10^{1}$, т. е. образуются полые частицы.

Важной характеристикой состояния вещества, связанной с трансформацией электронного спектра при переходе от массивного тела к низкоразмерным структурам, является ширина запрещенной зоны $\left(E_{g}\right)$. При синтезе субмикрочастиц $\mathrm{SrO}$ и $\mathrm{BaO}$ заданного размера, можно конструировать материалы с заранее известным электронным энергетическим спектром и требуемыми физическими свойствами.

Ширину запрещенной зоны частиц $\mathrm{SrO}$ и $\mathrm{BaO}$ определяли по спектрам диффузного отражения (СДО) и краю основной полосы оптического поглощения. Расчет проводили путем экстраполяции на ось абсцисс участка спектра в области резкого изменения коэффициента поглощения (рис. 3). Для полученных частиц оксида стронция и оксида бария оптическая ширина запрещенной зоны находится в диапазонах 3.15-3.19 эВ и 2.95-3.16 эВ соответственно (табл. 1), что сопоставимо с величиной данной характеристики для нанопленок на ос-

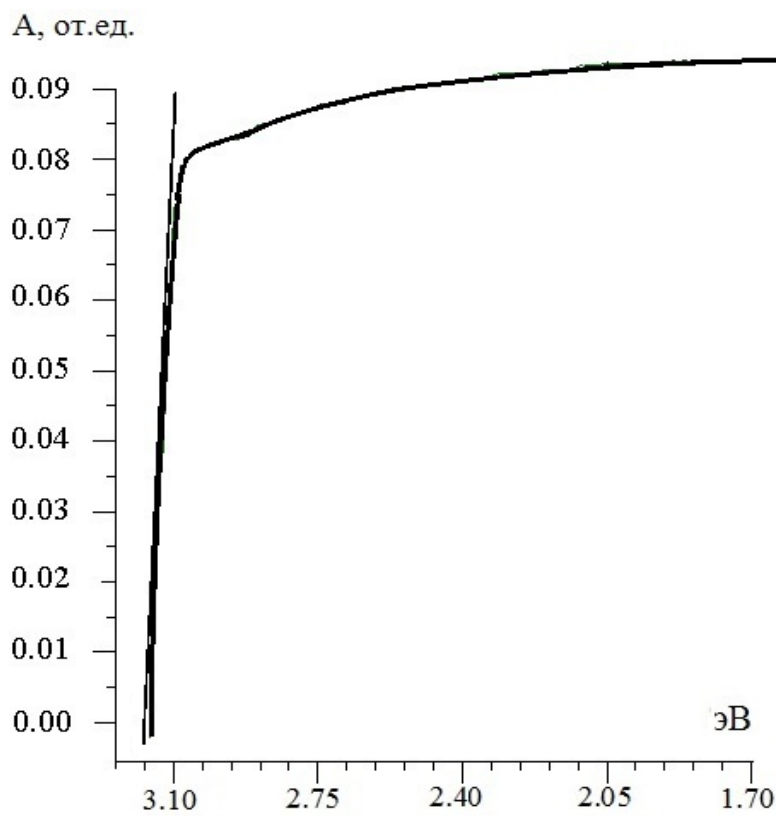

Рис. 3. Спектр диффузного отражения частц $\mathrm{SrO}$ $\left(\mathrm{C}_{\mathrm{Sr}^{\left(\mathrm{NO}_{3}\right)_{2}}}=3 \cdot 10^{-2} \mathrm{M}\right)$

[Fig. 3. Diffuse reflection spectrum of $\mathrm{SrO}$ particles

$$
\left.\left(\mathrm{C}_{\mathrm{Sr}_{\left(\mathrm{NO}_{3}\right)_{2}}}=3 \cdot 10^{-2} \mathrm{M}\right)\right]
$$

нове оксидов изучаемых металлов [19]. При уменьшении диаметра частиц нами обнаружено незначительное смещение края поглощения в СДО-спектрах в коротковолновую область, т. е. увеличения оптической ширины запрещенной зоны.

\section{ЗАКЛЮЧЕНИЕ}

Таким образом, в работе синтезированы субмикрочастицы $\mathrm{SrO}$ и $\mathrm{BaO}$ методом термического разложения аэрозоля, содержащего в качестве прекурсоров нитраты бария и стронция. Природа синтезируемых соединений доказана методом ИК-спектроскопии. Определены характерные линейные размеры и изучена морфология поверхности частиц методом атомно-силовой микроскопии. Анализ полученных АСМ-кадров показывает, что образующиеся частицы имеют форму близкую к сферической, размер частиц колеблется в диапазоне 115-560 нм в зависимости от исходной концентрации прекурсоров. Показано, что при пиролизе происходит дробление капли жидкости, а образующаяся твердая фаза представляет собой полые сферы. На основе спектров диффузного отражения определена оптическая ширина запрещенной зоны объектов, которая находится в диапазонах 3.15-3.19 эВ и 2.95-3.16 эВ для $\mathrm{SrO}$ и $\mathrm{BaO}$ соответственно. Полученные данные представляют интерес при дальнейшем изучении свойств суб- 
микро- и наноструктур на основе $\mathrm{SrO}$ и $\mathrm{BaO}$, а также при целенаправленном синтезе новых функциональных материалов, обладающих улучшенным комплексом свойств.

\section{СПИСОК ЛИТЕРАТУРЫ}

1. Noguera C. Physics and Chemistry at Oxide Surfaces. Cambridge University Press: Cambridge, U.K, 1996, $223 \mathrm{p}$.

2. Koumoto K., Terasaki I., Murayama N. Oxide Thermoelectrics. Research Signpost Trivandrum, India, 2002, $255 \mathrm{p}$.

3. Минакова Т. С., Екимова И. А. Фториды и оксиды щелочноземельных металлов и магния. Поверхностные свойства. Томск: Изд-й Дом ТГУ, 2014. 148 с.

4. Joon L. B., Jung K. H., Jeong W. I., Kim J. J. // Solar Energy Materials and Solar Cells, 2010, vol. 94, iss. 3, pp. 542-546. DOI: https://doi.org/10.1016/j.solmat.2009. 11.021

5. Чайникова А. С., Ваганова М. Л., Щеголева Н. Е., Лебедева Ю. Е. Технологические аспекты создания радиопрозрачных стеклокристаллических материалов на основе высокотемпературных алюмосиликатных систем (обзор) // Труды ВИАМ, 2015, № 11, с. 26-39.

6. Суздалев И. П. Электрические и магнитные переходы в нанокластерах и наноструктурах. М.: КРАСАНД, 2012, $480 \mathrm{c}$.

7. Gurav A., Kodas T., Pluym T., Xiong Y. // Aerosol Science and Technology, 1993, vol. 19, iss. 4, pp. 411-452. DOI: https://doi.org/10.1080/02786829308959650

8. Сергеева А. В., Наумов А. В., Семенов В. Н. // Конденсированные среды и межфазные границы, 2016, т .18, № 3, c. 367-373. http://www.kcmf.vsu.ru/resources/ t_18_3_2016_007.pdf

9. Hinklin T. R., Rand S. C., Laine R. M. // Adv. Mater, 2008, vol. 20, pp. 1270-1273. DOI: https://doi.org/10.1002/ adma.200701235
10. Jinsub Choi. Fabrication of Monodomain Porous Alumina using Nanoimprint Lithography and its Applications. Dissertation zur Erlangung des akademischen Grades Doktor-Ingenieur (Dr.-Ing.) vorgelegt an der MathematischNaturwissenschaftlich-Technischen Fakultät der MartinLuther-Universität Halle-Wittenberg verteidigt am 05.02.2004.

11. Лидин Р. А., Молочко В. А., Андреева Л. Л. Рeакции неорганических веществ: Справочник. 2-е изд., перераб. и доп. М.: Дрофа, 2007, 637 с.

12. Lashanizadegan M., Mousavi F., Mirzazadeh H. // J. of Ceram. Proces. Res., 2016, vol. 17, №. 6, pp. 586590 .

13. Zeenath Bazeera A., Irfana Amrin M. Synthesis and characterization of barium oxide nanoparticles // IOSR Journal of Applied Physics, 2017, pp. 76-80. DOI: https:// doi.org/10.9790/4861-17002017680

14. Richard A. Nyquist, Ronald O. Kagel. Handbook of Infrared and Raman Spectra of Inorganic Compounds and Organic Salts. 1971, Academic Press, New York and London, 499 p. DOI: https://doi.org/10.1016/C2009-0-22109-X

15. Пискунов М. В., Стрижак П. А. // Письма в ЖТФ, 2017, т. 43, № 12, с. 34-41. DOI: https://doi.org/10.21883/ PJTF.2017.12.44706.16709

16. Жуков А. С., Архипов В. А., Бондарчук С. С., Гольдин В. Д. // Химическая физика, 2013, т. 32, № 12, c. 52-58. DOI: https://doi.org/10.7868/S0207401X1 3120108

17. Лыков А. В. Теория теплопроводности. М.: Высш. шк., 1967, 600 с.

18. Померанцев В. В., Арефьев К. М., Ахмедов Д. Б. и др. Основы практической теории горения. М.: Энергия, 1986, $312 \mathrm{c.}$

19. Abdulazeez O. Mousa, Noor A. Nema and Hasan H. Hasan. // Journal of Chemical and Pharmaceutical Research, 2016, vol. 8(8), pp. 832-840. http://www.jocpr.com/articles/ effect-of-annealing-on-barium-oxide-bao-thin-films-prepared-by-chemical-spray-pyrolysis-csp-technique.pdf

\title{
SYNTHESIS OF SrO AND BaO SUBMICROPARTICLES BY THE METHOD OF THERMAL DECOMPOSITION OF AEROSOLS
}

\author{
(C) 2018 A. I. Gorshkov, E. N. Gribanov, E. R. Oskotskaya \\ Turgenev Orel State University, 95 Komsomolskaya str., 302026 Orel, Russia \\ e-mail: gribanovEN@gmail.com
}

Received 06.11.2018

\begin{abstract}
In the paper submicroparticles of strontium oxide and barium oxide were obtained by thermal decomposition of aerosols. The nature of the synthesized compounds was proved by IR spectroscopy. The characteristic linear dimensions were determined and the surface morphology of the particles was studied by atomic force microscopy. Analysis of the obtained AFM-frames shows that the formed particles have a shape close to spherical, and their size varies in the range of 115$560 \mathrm{~nm}$, depending on the initial concentration of precursors. It is shown that during the pyrolysis
\end{abstract}


the splitting of a drop of liquid occurs, and the resulting solid phase is hollow spheres. The optical band gap of the particles of strontium and barium oxides was determined on the basis of their diffuse reflection spectra (DRS) along the edge of the main optical absorption band. It is in the ranges of $3.15-3.19 \mathrm{eV}$ and $2.95-3.16 \mathrm{eV}$, respectively. We found a manifestation of the size effect associated with the interrelation of reducing the diameter of $\mathrm{SrO}$ and $\mathrm{BaO}$ particles and the narrowing of the characteristic absorption bands in the IR spectra, as well as the shift of the absorption edge in the DRS-spectra to the shortwave region, i.e. increasing the optical band gap. The data obtained are of interest in the further study of the properties of submicro- and nanostructures based on strontium oxide and barium oxide, as well as in the synthesis of new functional materials with improved complex properties.

Keywords: strontium oxide, barium oxide, band gap, pyrolysis.

DOI: https://doi.org/10.17308/kcmf.2018.20/631

\section{REFERENCES}

1. Noguera C. Physics and Chemistry at Oxide Surfaces. Cambridge University Press: Cambridge, U.K., 1996, $223 \mathrm{p}$.

2. Koumoto K., Terasaki I., Murayama N. Oxide Thermoelectrics. Research Signpost Trivandrum, India, 2002, $255 \mathrm{p}$.

3. Minakova T. S., Ekimova I. A. Fluorides and Oxides of Alkaline Earth Metals. Surface Property. Tomsk, Publ. House of Tomsk State University, 2014, 148 p. (in Russ.)

4. Joon L. B., Jung K. H., Jeong W. I., Kim J. J. Solar Energy Materials and Solar Cells, 2010, vol. 94, iss. 3, pp. 542-546. DOI: https://doi.org/10.1016/j.solmat.2009. 11.021

5. Chaynikova S. A., Vaganova M. L., Shchegoleva. N. E., Lebedeva E. Yu. Technological Aspects of the Creation of High-temperature Radio-transparent Glass-crystal Materials Based on Aluminosilicate Systems (Review). Trudy VIAM, 2015, no. 11, pp. 26-39. (in Russ.)

6. Suzdalev I. P. Electrical and Magnetic Transitions in Nanoclusters and Nanostructures. Moscow, KRASANG Publ., 2012, 480 p. (in Russ.)

7. Gurav A., Kodas T., Pluym T., Xiong Y. Aerosol Science and Technology, 1993, vol. 19, iss. 4, pp. 411-452. DOI: https://doi.org/10.1080/02786829308959650

8. Sergeeva V. A., Naumov A. V., Semenov V. N. Condensed Matter and Interphase, 2016.,vol. 18., no. 3, pp. 367-373. Available at: http://www.kcmf.vsu.ru/ resources/t_18_3_2016_007.pdf (in Russ.)

9. Hinklin T. R., Rand S. C., Laine R. M. Adv. Mater., 2008, vol. 20, pp. 1270-1273. DOI: https://doi.org/10.1002/ adma.200701235

10. Jinsub Choi. Fabrication of Monodomain Porous Alumina using Nanoimprint Lithography and its Applications. Dissertation zur Erlangung des akademischen Grades Doktor-Ingenieur (Dr.-Ing.) vorgelegt an der Mathematisch-
Naturwissenschaftlich-Technischen Fakultät der MartinLuther-Universität Halle-Wittenberg verteidigt am 05.02.2004.

11. Lidin V. A., Molochko L. A., Andreeva L. L. Reactions of Inorganic Substances: Handbook of 2nd ed., Rev. and add. Moscow, Drofa Publ., 2007, 637 p. (in Russ.)

12. Lashanizadegan M., Mousavi F., Mirzazadeh H. Journal of Ceramic Process. Research, 2016, vol. 17, no. 6, pp. 586-590.

13. Zeenath Bazeera A., Irfana Amrin M. IOSR Journal of Applied Physics, 2017, pp. 76-80. DOI: https://doi. org/10.9790/4861-17002017680

14. Richard A. Nyquist, Ronald O. Kagel. Handbook of Infrared and Raman Spectra of Inorganic Compounds and Organic Salts. 1971, Academic Press, New York and London, 499 p. DOI: https://doi.org/10.1016/C2009-022109-X

15. Piskunov M. V., Strizhak P. A. Technical Physics Letters, 2017, vol. 43, no. 6, pp. 558-561. DOI: https://doi. org/10.1134/S1063785017060256

16. Zhukov A. S., Gol'Din V. D., Arkhipov V. A., Bondarchuk S. S. Russian Journal of Physical Chemistry B., 2013, vol. 7, no. 6, pp. 777-782. DOI: https://doi. org/10.1134/S199079311306016X

17. Lykov A. V. The theory of Thermal Conductivity. Moscow, Vysshaya Shkola Publ., 1967, 600 p. (in Russ.)

18. Pomerantsev V. V., Aref'ev K. M., Akhmedov D. B. Fundamentals of Applied Theory of Combustion. Moscow, Energy Publ, 1986. 312 p. (in Russ.)

19. Abdulazeez O. Mousa, Noor A. Nema and Hasan H. Hasan. Journal of Chemical and Pharmaceutical Research, 2016, no. 8, pp. 832-840. Available at: http:// www.jocpr.com/articles/effect-of-annealing-on-bariumoxide-bao-thin-films-prepared-by-chemical-spraypyrolysis-csp-technique.pdf 


\section{А. И. ГОРШКОВ, Е. Н. ГРИБАНОВ, Э. Р. ОСКОТСКАЯ}

Гориков Александр Игоревич - аспирант кафедры химии, Орловский государственный университет имени И. С. Тургенева, Орел, Россия; e-mail: gribanovEN@gmail.com

Грибанов Евгений Николаевич - к. х. н., доцент кафедры химии, Орловский государственный университет имени И. С. Тургенева, Орел, Россия; e-mail: gribanovEN@gmail.com

Оскотская Эмма Рафаиловна - д. х. н., профессор, зав. кафедрой химии, Орловский государственный университет имени И. С. Тургенева, Орел, Россия
Aleksandr I. Gorshkov - Graduate Student, Department of Chemistry, Turgenev Orel State University, Orel, Russia; e-mail: gribanovEN@gmail.com

Eugene N. Gribanov - Cand. Sci. (Chem.), Associate Professor of the Department of Chemistry, Turgenev Orel State University, Orel, Russia; e-mail: gribanovEN@gmail.com

Emma R. Osckotskaya - Dr, Sci, (Chem.), Professor, Head of the Department of Chemistry, Turgenev Orel State University, Orel, Russia 